\title{
The Riemann Zeta Function on Arithmetic Progressions
}

\author{
Jörn Steuding and Elias Wegert
}

\section{CONTENTS}

1. Introduction

2. Proof of the Main Theorem

3. Concluding Remarks

References

2000 AMS Subject Classification: Primary 11M06

Keywords: Riemann zeta function, value distribution, arithmetic progression
We prove asymptotic formulas for the first discrete moment of the Riemann zeta function on certain vertical arithmetic progressions inside the critical strip. The results give some heuristic arguments for a stochastic periodicity that we observed in the phase portrait of the zeta function.

\section{INTRODUCTION}

The Riemann zeta function $\zeta(s)$ is of special interest in number theory and complex analysis. The real zeros of $\zeta(s)$ are located at $s=-2 n, n \in \mathbb{N}$, and are said to be trivial. All other zeros are called nontrivial and lie inside the critical strip $0<\operatorname{Re} s<1$. The famous Riemann hypothesis claims that all nontrivial zeros lie on the critical line $\operatorname{Re} s=1 / 2$.

We are interested in the values of the zeta function on certain vertical arithmetic progressions. In [Putnam 54a, Putnam 54b], it was shown that there exists no infinite arithmetic progression of nontrivial zeros, and in [Frankenhuijsen 05], the author obtained bounds for the length of any hypothetical arithmetic progression. Asymptotic formulas proved for the second and fourth moments of the Riemann zeta function on arbitrary arithmetic progressions to the right of the critical line were proved in [Good 78]. Here we shall investigate the discrete first moments

$$
A(s, d, M):=\frac{1}{M} \sum_{0 \leq m<M} \zeta(s+\mathrm{i} m d)
$$

for positive real numbers $d$. It turns out that for special values of $d>0$, the asymptotic behavior of $A(s, d, M)$ for $M \rightarrow \infty$ can be described explicitly.

Theorem 1.1. Fix $s \in \mathbb{C} \backslash\{1\}$ with $0<\sigma:=\operatorname{Re} s \leq 1$, $t:=\operatorname{Im} s \geq 0$, and let $d=2 \pi / \log \ell$, where $\ell \geq 2$ is an integer. Then for $M \rightarrow+\infty$,

$$
\frac{1}{M} \sum_{0 \leq m<M} \zeta(s+\mathrm{i} m d)=\frac{1}{1-\ell^{-s}}+O\left(M^{-\sigma} \log M\right) .
$$




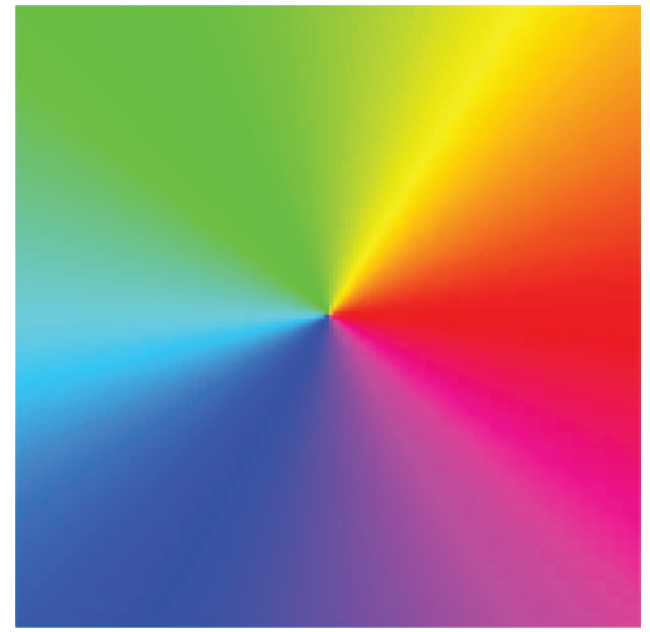

FIGURE 1. A phase portrait of $f(z)=z$ (color figure available online).

We would like to mention that our interest in sums of the Riemann zeta function on arithmetic progressions has also been motivated by phase portraits.

The phase portrait (or phase plot) of a meromorphic function $f: D \rightarrow \widehat{\mathbb{C}}$ visualizes $f$ directly on its domain $D$ by color-coding its phase $\psi:=f /|f|$. In Figure 1 , the standard hsv color scheme is used to encode the phase of all points in a square (with the origin at its center), which yields the phase portrait of $f(z)=z$.

For an introduction to phase plots and related phase diagrams we refer to [Wegert and Semmler 11] and [Wegert 10], respectively. Here we just point out that meromorphic functions can be reconstructed from their phase portraits uniquely up to a positive scaling factor. Figure 2 shows the phase plot of the Riemann zeta function in the square $-40 \leq \operatorname{Re} z \leq 10,-2 \leq \operatorname{Im} z \leq 48$.

Visual inspection of the phase plot of $\zeta$ in the critical strip almost immediately reveals a surprising "stochastic periodicity" of the phase $\psi=\zeta /|\zeta|$. This is illustrated in Figure 3, which shows 20 consecutive rectangles of width 1 and height 20 from the critical strip, with a small overlapping region between neighboring domains.

The eye-catching yellow diagonal stripes led us to the conjecture that the phase plot of zeta in the critical strip has a sort of stochastic period, and some heuristic arguments suggest that its length equals $2 \pi / \log 2$. One option to make this statement more precise is to study mean values of the phase on arithmetic progressions,

$$
\frac{1}{M} \sum_{0 \leq m<M} \frac{\zeta(s+\mathrm{i} m d)}{|\zeta(s+\mathrm{i} m d)|}
$$

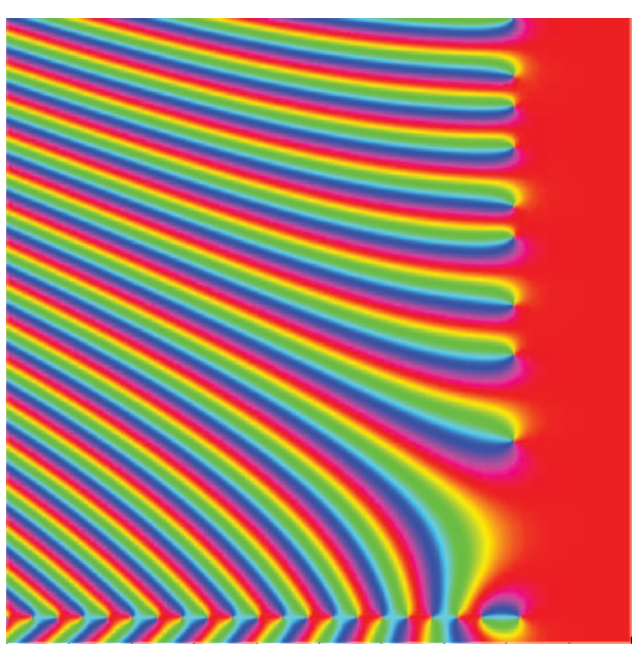

FIGURE 2. A phase portrait of the Riemann zeta function (color figure available online).

If the phase is randomly distributed (on the unit circle), these mean values should be almost independent of the choice of $d$ and $s$ for sufficiently large $M$. On the other hand, a periodic color pattern should manifest itself in the behavior of the mean values if $d$ is in resonance with the period.

The investigation of (1-2) seems to be a hard problem, and we decided to omit the normalization of the summands, i.e., to investigate (1-1) instead of (1-2), hoping that the latter sums reflect the behavior of the former. This is, in a sense, confirmed by Theorem 1.1. If $d=2 \pi / \log \ell$ with $\ell \in \mathbb{Z}$ and $\ell \geq 2$, then there is indeed an asymptotic periodicity of $s \mapsto A(s, d, M)$ for large $M$. Moreover, among all values of $\ell$, the strongest effect comes from $\ell=2$, which coincides with our observations.

\section{PROOF OF THE MAIN THEOREM}

In what follows, we consider $d>0$ and $s \in \mathbb{C}$ with $0<$ $\sigma:=\operatorname{Re} s<1, t:=\operatorname{Im} s \geq 0$ as being fixed. The basic tool

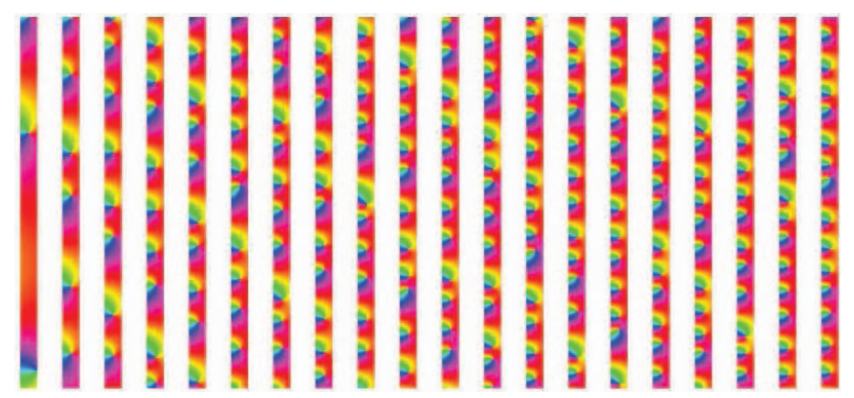

FIGURE 3. Phase plots of the Riemann zeta function along the critical strip (color figure available online). 
in proving Theorem 1.1 is the representation

$$
\zeta(s)=\frac{x^{1-s}}{s-1}+\sum_{n \leq x} n^{-s}+O\left(x^{-\sigma}\right)
$$

valid for any $s$ with $0<\sigma \leq 2$ and $x \geq t / \pi$ (see [Ivić 85, Theorem 1.8]). Note that the $O\left(x^{-\sigma}\right)$ term is uniform with respect to $t$ and $\sigma$ for $|t| \leq \pi x$ and $0<\sigma_{0} \leq \sigma \leq 2$. Evaluating the sums (1-1) with the asymptotic formula $(2-1)$, we obtain

$$
\begin{aligned}
M \cdot A(s, d, M)= & \sum_{0 \leq m<M} \frac{x^{1-s-\mathrm{i} m d}}{s+\mathrm{i} m d-1} \\
& +\sum_{0 \leq m<M} \sum_{n \leq x} n^{-s-\mathrm{i} m d}+O\left(M x^{-\sigma}\right),
\end{aligned}
$$

where $x \geq(t+d M) / \pi$. A straightforward estimate yields

$$
\begin{aligned}
\left|\sum_{0 \leq m<M} \frac{x^{1-s-\mathrm{i} m d}}{s+\mathrm{i} m d-1}\right| & \leq x^{1-\sigma}\left(\frac{1}{\sigma}+\sum_{1 \leq m<M} \frac{1}{t+m d}\right) \\
& =O\left(x^{1-\sigma} \log M\right) .
\end{aligned}
$$

In the next step we fix $M, N \in \mathbb{N}$ and study the double sums

$$
\Sigma_{N, M}(s):=\sum_{1 \leq n \leq N} \sum_{0 \leq m<M} n^{-s-\mathrm{i} m d} .
$$

In order to estimate the inner sums, we introduce $\Delta:=d /(2 \pi)$ and remark that $n^{-\mathrm{i} d}=\mathrm{e}^{2 \pi \mathrm{i} \alpha_{n}}$ or $n^{-\mathrm{i} d}=$ $\mathrm{e}^{-2 \pi \mathrm{i} \alpha_{n}}$, where $\alpha_{n}:=\|\Delta \log n\|$ denotes the distance of $\Delta \log n$ to the integers. Setting $b:=\mathrm{e}^{1 / \Delta}$, we have

$$
\alpha_{n}=\min _{j \in \mathbb{Z}}|j-\Delta \log n|=\Delta \min _{j \in \mathbb{Z}}\left|\log \left(n b^{-j}\right)\right| .
$$

The outer sum is split into three parts by grouping the summands according to the values of $\alpha_{n}$. A positive integer $n$ is said to be special if it belongs to the set

$$
\mathcal{S}:=\left\{n \in \mathbb{N}: \min _{j \in \mathbb{Z}}\left|n-b^{j}\right|<\frac{1}{2}\right\} .
$$

The remaining integers in $\mathcal{N}:=\mathbb{N} \backslash \mathcal{S}$ are called normal. The set $\mathcal{S}$ is the disjoint union of $\mathcal{S}_{0}$ and $\mathcal{S}_{+}$,

$$
\mathcal{S}_{0}:=\left\{n \in \mathcal{S}: \alpha_{n}=0\right\}, \quad \mathcal{S}_{+}:=\left\{n \in \mathcal{S}: \alpha_{n}>0\right\} .
$$

Note that we always have $1 \in \mathcal{S}_{0}$. Further, $\mathcal{S}_{0} \neq\{1\}$ if and only if $\Delta=k / \log l$ with $k, l \in \mathbb{Z}$ and $l \geq 2$, i.e., $d$ belongs to the exceptional set

$$
E:=\left\{d \in \mathbb{R}_{+}: d=\frac{2 k \pi}{\log l}, k, l \in \mathbb{Z}, l \geq 2\right\} .
$$

According to the decomposition of $\mathbb{N}$ we have

$$
\begin{aligned}
& \Sigma_{N, M}(s)=\sum_{\substack{1 \leq n \leq N \\
n \in \mathcal{S}_{0}}} \sum_{\substack{0 \leq m<M \\
\cdots+}} \cdots \sum_{\substack{1 \leq n \leq N \\
n \in \mathcal{N}}} \sum_{0 \leq m<M} \cdots \\
& +\sum_{\substack{1 \leq n \leq N \\
n \in \mathcal{S}_{+}}} \sum_{\substack{0 \leq m<M \\
\cdots}} \cdots
\end{aligned}
$$

The first sum extends over those integers $n$ with $\alpha_{n}=0$ and can be computed explicitly. If $\mathcal{S}_{0}=\{1\}$, then

$$
\sum_{\substack{1 \leq n \leq N \\ n \in \mathcal{S}_{0}}} \sum_{0 \leq m<M} n^{-s-\mathrm{i} m d}=M
$$

If $\mathcal{S}_{0} \neq\{1\}$, we denote by $\ell$ the smallest integer $n$ with $n \geq 2$ and $\alpha_{n}=0$. Then $\alpha_{n}=0$ if and only if $n=\ell^{j}$ with $j \in \mathbb{N} \cup\{0\}$. Hence, with $J:=\lfloor\log N / \log \ell\rfloor+1$,

$$
\begin{aligned}
& \sum_{\substack{1 \leq n \leq N \\
n \in \mathcal{S}_{0}}} \sum_{0 \leq m<M} n^{-s-\mathrm{i} m d}=M \sum_{\substack{1 \leq n \leq N \\
\alpha n=0}} n^{-s} \\
& =M \sum_{0 \leq j \leq J-1} \ell^{-j s}=M \frac{1-\ell^{-s J}}{1-\ell^{-s}} \\
& =\frac{M}{1-\ell^{-s}}+O\left(M N^{-\sigma}\right) \text {. }
\end{aligned}
$$

The estimations of the second and the third sum in $(2-7)$ are based on the following lemma.

Lemma 2.1. Fix $\Delta:=d /(2 \pi)$ and $n \in \mathbb{N}$. If $\alpha_{n}=$ $\|\Delta \log n\| \neq 0$, then for all $M \in \mathbb{N}$,

$$
\left|\sum_{0 \leq m<M} \mathrm{e}^{-\mathrm{i} m d \log n}\right|<\frac{1}{2 \alpha_{n}}
$$

Proof. Recall that $\mathrm{e}^{-\mathrm{i} d \log n}=\mathrm{e}^{ \pm 2 \pi \mathrm{i} \alpha_{n}}$. Since $0<$ $2 \pi \alpha_{n} \leq \pi, /$ break it follows that $\left|1-\mathrm{e}^{ \pm 2 \pi \mathrm{i} \alpha_{n}}\right| \geq 4 \alpha_{n}$, and thus

$$
\begin{aligned}
\left|\sum_{0 \leq m<M} \mathrm{e}^{-\mathrm{i} m d \log n}\right| & =\left|\sum_{0 \leq m<M} \mathrm{e}^{ \pm 2 \pi \mathrm{i} m \alpha_{n}}\right| \\
& =\left|\frac{1-\mathrm{e}^{ \pm 2 \pi \mathrm{i} M \alpha}}{1-\mathrm{e}^{ \pm 2 \pi \mathrm{i} \alpha_{n}}}\right|<\frac{2}{4 \alpha_{n}} .
\end{aligned}
$$

The second sum in $(2-7)$ is estimated using Lemma 2.1,

$$
\left|\sum_{\substack{1 \leq n \leq N \\ n \in \mathcal{N}}} \sum_{0 \leq m<M} n^{-s-\mathrm{i} m d}\right| \leq \sum_{\substack{1 \leq n \leq N \\ n \in \mathcal{N}}} \frac{1}{2 n^{\sigma} \alpha_{n}} .
$$

Since $b=\mathrm{e}^{1 / \Delta}>1$, it follows that every nonnegative integer $n$ satisfies

$$
b^{j}-\frac{1}{2} \leq n<b^{j+1}-\frac{1}{2}
$$


for some natural number $j$. We denote by $k_{n}^{+}$and $k_{n}^{-}$the nearest integers to $n-b^{j}$ and $n-b^{j+1}$, respectively,

$$
k_{n}^{+}:=\left\lfloor n-b^{j}+1 / 2\right\rfloor, \quad k_{n}^{-}:=\left\lfloor n-b^{j+1}+1 / 2\right\rfloor .
$$

Then

$$
0 \leq k_{n}^{+} \leq b^{j+1}-b^{j}, \quad b^{j}-b^{j+1}-1 \leq k_{n}^{-} \leq-1,
$$

and $n$ can be represented as

$$
n=b^{j}+k_{n}^{+}+\mu_{n}, \quad n=b^{j+1}+k_{n}^{-}+\nu_{n},
$$

with $-1 / 2<\mu_{n}, \nu_{n} \leq 1 / 2$. To simplify notation we just write $\mu, \nu, k^{-}$, and $k^{+}$, respectively. From (2-5) we infer that $\alpha_{n} \log b$ is the minimum of the two numbers

$$
\left|\log \left(1+\frac{\mu+k^{+}}{b^{j}}\right)\right|, \quad\left|\log \left(1+\frac{\nu+k^{-}}{b^{j+1}}\right)\right| .
$$

Now it follows from $(2-12)$ that

$$
-\frac{1}{2 b^{j}} \leq \frac{\mu+k^{+}}{b^{j}}<b, \quad-\frac{1}{2 b^{j+1}}<\frac{\nu+k^{-}}{b^{j+1}}<0,
$$

and since $|\log (1+x)| \geq|x| /(1+a)$ for $-1<x \leq a$, we have

$$
\begin{aligned}
& \left|\log \left(1+\frac{\mu+k^{+}}{b^{j}}\right)\right| \geq \frac{\left|\mu+k^{+}\right|}{b^{j}(1+b)} \geq \frac{k^{+}}{2 b^{j}(1+b)} \geq \frac{k^{+}}{4 b^{j+1}}, \\
& \left|\log \left(1+\frac{\nu+k^{-}}{b^{j+1}}\right)\right| \geq \frac{\left|\nu+k^{-}\right|}{b^{j+1}} \geq \frac{\left|k^{-}\right|}{2 b^{j+1}} .
\end{aligned}
$$

Consequently,

$$
\alpha_{n} \geq \frac{\min \left\{\left|k_{n}^{-}\right|, k_{n}^{+}\right\}}{4 b^{j+1} \log b} .
$$

As $n \in \mathbb{N}$ runs through the interval $\left[b^{j}-1 / 2, b^{j+1}\right.$ $-1 / 2), k_{n}^{-}$attains every integer in $\left[b^{j}-b^{j+1}-1,-1\right]$ at most once, and $k_{n}^{+}$attains every integer in $\left[1, b^{j+1}-b^{j}\right]$ at most once.

Setting $J:=\lfloor\log N / \log b\rfloor+1$ and using (2-11), $(2-14)$, and $n \geq b^{j}-1 / 2 \geq b^{j} / 2$, we estimate the second sum on the right-hand side of $(2-7)$ for all $N \geq$ $\max \{3, b\}$

$$
\begin{aligned}
& \left|\sum_{\substack{1 \leq n \leq N \\
n \in \mathcal{N}}} \sum_{0 \leq m<M} n^{-s-\mathrm{i} m d}\right| \\
& \quad \leq \sum_{\substack{1 \leq n \leq N \\
n \in \mathcal{N}}} \frac{1}{2 n^{\sigma} \alpha_{n}} \leq \sum_{0 \leq j \leq J} \sum_{1 \leq k<b^{j+1}} \frac{8 b^{j+1} \log b}{b^{j \sigma} k} \\
& \quad \leq 8 b \log b \sum_{0 \leq j<J} b^{(1-\sigma) j} \sum_{1 \leq k<b^{j+1}} \frac{1}{k} \\
& \quad \leq 8 b \log b \sum_{0 \leq j<J} b^{(1-\sigma) j}(1+(j+1) \log b) \\
& \quad \leq \frac{8 b \log b}{b^{1-\sigma}-1}\left(b^{J(1-\sigma)}(J \log b+1)\right) \\
& \quad=O\left(N^{1-\sigma} \log N\right) .
\end{aligned}
$$

Before we study the last sum of $(2-7)$ over $n \in \mathcal{S}_{+}$, we summarize the results.

Lemma 2.2. Let $d>0, s=\sigma+\mathrm{i} t \neq 1$ with $0<\sigma<1$ and $t \geq 0$, and set

$$
\Sigma_{N, M}(s):=\sum_{1 \leq n \leq N} \sum_{0 \leq m<M} n^{-s-\mathrm{i} m d} .
$$

(i) If $d$ does not belong to the exceptional set $E$ defined in $(2-6)$, then

$$
\begin{aligned}
\Sigma_{N, M}(s)= & M+\sum_{\substack{1 \leq n \leq N \\
n \in \mathcal{S}_{+}}} \sum_{0 \leq m<M} n^{-s-\mathrm{i} m d} \\
& +O\left(N^{1-\sigma} \log N\right) .
\end{aligned}
$$

(ii) If $d \in E$ and $\ell \geq 2$ is the smallest integer with $d=$ $2 k \pi / \log \ell$, then

$$
\begin{aligned}
\Sigma_{N, M}(s)= & \frac{M}{1-\ell^{-s}}+\sum_{\substack{1 \leq n \leq N \\
n \in \mathcal{S}_{+}}} \sum_{0 \leq m<M} n^{-s-\mathrm{i} m d} \\
& +O\left(N^{-\sigma}(M+N \log N)\right) .
\end{aligned}
$$

(iii) If $d=2 \pi / \log \ell$ with $\ell \in \mathbb{N}$ and $\ell \geq 2$, then

$$
\Sigma_{N, M}(s)=\frac{M}{1-\ell^{-s}}+O\left(N^{-\sigma}(M+N \log N)\right) .
$$

Proof. The first two statements follow from (2-8), (2-9), and $(2-15)$. If $d=2 \pi / \ell$, then $\Delta=1 / \log \ell$ and $\alpha_{n}=0$ if and only if $n=\ell^{p}$ for some $p \in \mathbb{N}$. Moreover, if $\alpha_{n} \neq 0$, then $\left|n-\ell^{j}\right| \geq 1$ for all $j \in \mathbb{Z}$, i.e., $\mathcal{S}_{+}$is void.

To complete the proof of Theorem 1.1 it suffices to choose $x=N=\lfloor M d+t\rfloor$ and to apply the asymptotic formula $(2-1)$ to $\zeta(s+\mathrm{i} m d)$. This yields $(2-2)$, and the desired result then follows from (2-3) and Lemma 2.2(iii). 
In the cases that are not covered by Theorem 1.1, the behavior of the sum over $n \in \mathcal{S}_{+}$is crucial. Again we have the estimate

$$
\left|\sum_{\substack{1 \leq n \leq N \\ n \in \mathcal{S}_{+}}} \sum_{0 \leq m<M} n^{-s-\mathrm{i} m d}\right| \leq \sum_{\substack{1 \leq n \leq N \\ n \in \mathcal{S}_{+}}} \frac{1}{2 n^{\sigma} \alpha_{n}},
$$

but even in the case that $\Delta=k / \log \ell$ with $k \geq 2$ (and the smallest possible value of $\ell$ ), the sum on the righthand side is difficult to estimate. If, for example, there exists a fixed value $c$ such that $1 \leq\left|n^{k}-\ell^{j}\right| \leq c$ for an infinite number of positive integers $n$ and $j$, then

$$
\alpha_{n} \sim \frac{\Delta}{k}\left|\log \left(1 \pm c \ell^{-j}\right)\right| \sim \frac{c \Delta}{k} \ell^{-j} \sim \frac{c \Delta}{k} n^{k} .
$$

Consequently, if the considered sum admits an estimate

$$
\sum_{\substack{1 \leq n \leq N \\ n \in \mathcal{S}_{+}}} \frac{1}{2 n^{\sigma} \alpha_{n}} \leq C N^{\beta}
$$

then $\beta \geq k-\sigma$, which is useless for $k \geq 2$.

\section{CONCLUDING REMARKS}

The asymptotic formula of Theorem 1.1 holds in a wider range, namely the right half-plane $\operatorname{Re} s>0$, provided that no term of the arithmetic progression $s+\mathrm{i} m d$ is identical to the pole of the zeta function at 1 . Actually, in the half-plane $\operatorname{Re} s>1$, where the zeta function is given by an absolutely convergent Dirichlet series,

$$
\zeta(s)=\sum_{n=1}^{\infty} \frac{1}{n^{s}}
$$

the asymptotic behavior of $A(s, d, M)$ can be found quite easily for all $d$ in the exceptional set $E$. Using the same notation as above, we have, for some positive integer $N$,

$$
\begin{aligned}
& \sum_{0 \leq m<M} \zeta(s+\mathrm{i} m d) \\
& \quad=M \sum_{\substack{n \\
n \in \mathcal{S}_{0}}} n^{-s}+\left\{\sum_{\substack{n<N \\
n \notin \mathcal{S}_{0}}}+\sum_{\substack{n \geq N \\
n \notin \mathcal{S}_{0}}}\right\} n^{-s} \sum_{0 \leq m<M} \mathrm{e}^{-\mathrm{i} m d \log n} \\
& \quad=S_{1}+S_{2}+S_{3},
\end{aligned}
$$

say. The first sum $S_{1}$ yields the main term as in the proof of Theorem 1.1. In view of Lemma 2.1, the sum over $m$ in $S_{2}$ is bounded by $1 / \min _{n<N} \alpha_{n}$, and thus $S_{2}$ is bounded by some constant independent of $M$. For $S_{3}$ we use the trivial bound to obtain

$$
S_{3} \ll M \sum_{n \geq N} n^{-\operatorname{Re} s} \ll M N^{1-\operatorname{Re} s} .
$$

Since $\operatorname{Re} s>1$, this implies

$$
\frac{1}{M} \sum_{0 \leq m<M} \zeta(s+\mathrm{i} m d)=C(s, d)+o(1)
$$

as $M \rightarrow \infty$, where $C(s, d)=\left(1-\ell^{-s}\right)^{-1}$ for $d \in E$, and $C(s, d)=1$ otherwise. For Re $s=1$, the proof of Theorem 1.1 has to be modified in an obvious way.

As mentioned in the introduction, asymptotic formulas were obtained in [Good 78] for the second and fourth moments of the zeta function on arbitrary vertical arithmetic progressions for $\operatorname{Re} s>1 / 2$, for instance,

$$
\frac{1}{M} \sum_{0 \leq m<M}|\zeta(\sigma+\mathrm{i} m d)|^{4}=\sum_{n=1}^{\infty} \frac{d(n)^{2}}{n^{2 \sigma}}+o(1)
$$

as $M \rightarrow \infty$, provided that $d$ is not of the form $2 \pi l\left(\log \frac{m}{n}\right)^{-1}$ with integral $l \neq 0$ and positive integers $m \neq n$. Here the coefficients of the Dirichlet series on the right-hand side are defined by the divisor function $d(n):=\sum_{d \mid n} 1$; hence the series on the right does not converge for $\sigma=1 / 2$. Good's approach yields asymptotic formulas with remainder term if the common difference $d$ of the arithmetic progression is of special Diophantine type. Applying the Cauchy-Schwarz inequality, we see that the results of [Good 78] can be used to show that the first moment $A(s, d, M)$ is bounded as $M \rightarrow \infty$ for every value of $d$, provided that $\sigma>1 / 2($ and $s+\mathrm{i} m d \neq 1$ ).

In contrast to Good's results, Theorem 1.1 provides an asymptotic formula for the whole critical strip $0<$ Re $s<1$ for specific values of $d$.

\section{REFERENCES}

[Borwein et al. 07] P. Borwein, S. Choi, B. Rooney, and A. Weirathmueller (editors). The Riemann Hypothesis. A resource for the Afficionado and Virtuoso Alike. Springer, 2007.

[Frankenhuijsen 05] M. van Frankenhuijsen. "Arithmetic Progressions of Zeros of the Riemann Zeta Function." J. Number Theor. 115 (2005), 360-370.

[Good 78] A. Good. "Diskrete Mittel für einige Zetafunktionen." J. Reine Angew. Math. 303/304 (1978), 51-73.

[Ivić 85] A. Ivić. The Riemann Zeta Function. John Wiley \& Sons, 1985.

[Putnam 54a] C. R. Putnam. "On the Non-periodicity of the Zeros of the Riemann Zeta Function." Amer. J. Math. 76 (1954), 97-99. 
[Putnam 54b] C. R. Putnam. "Remarks on Periodic Sequences and the Riemann Zeta Function." Amer. J. Math. 76 (1954), 828-830.

[Wegert 10] E. Wegert. "Phase Diagrams of Meromorphic Functions." Comput. Methods Funct. Theory 10:2 (2010), 639-661.
[Wegert and Semmler 11] E. Wegert and G. Semmler. "Phase Plots of Complex Functions: A Journey in Illustration." Notices of the AMS 58 (2011) $768-780$.

Jörn Steuding, Department of Mathematics, Würzburg University, Am Hubland, 97218 Würzburg, Germany (steuding@mathematik.uni-wuerzburg.de)

Elias Wegert, Institute of Applied Analysis, TU Bergakademie Freiberg, 09596 Freiberg, Germany (wegert@math.tu-freiberg.de) 